\title{
Antioxidant Properties of Polyphenols and Their Potential Use in Improvement of Male Fertility: A Review
}

\author{
Yasmina Seddiki, Helena Moreira da Silva and Fernando Moreira da Silva* \\ University of the Azores, Faculty of Agrarian Sciences and Environment Animal Reproduction, Portugal
}

Received: July 27, 2017; Published: August 07, 2017

*Corresponding author: Fernando Moreira da Silva, University of the Azores, Faculty of Agrarian Sciences and Environment Animal Reproduction, 9700 Angra do Heroismo, Portugal

\begin{abstract}
Recent studies have investigated the use of plant bioactive substances, such as polyphenols, as antioxidants in assisted reproduction technologies. Semen cryopreservation is a technique that can affect sperm quality. Indeed, cryopreservation of human semen produces reactive oxygen species (ROS), which may cause damage in sperm cells. Antioxidants have been used in a number of studies to counter oxidative stress, both as dietary supplements and as components of culture media for in vitro experiments. Quercetin, catechin and resveratrol are three polyphenols produced by plants that exhibit antioxidant properties. Research has shown that these three compounds have the ability to increase antioxidant activity in human or animal semen. The studies reported in this review show positive and negative effects of the polyphenols, especially at high doses. More research is required to understand the cellular mechanisms involved in antioxidant activity and to examine the dose-dependent effects of polyphenols in semen cryopreservation.
\end{abstract}

Keywords: Antioxidants; Semen; Fertility; Quercetin; Catechin; Resveratrol

Abbreviations: IVF: In Vitro Fertilization; ROS: Reactive Oxygen Species; EGCG: Epigallocatechin Gallate; ECG: Epicatechin Gallate; EC: Epicatechin; EGC: Epigallocatechin; TCM: Tissue Culture Medium

\section{Introduction}

There has been a growing interest in the use of plant bioactive substances, such as polyphenols, as antioxidants in assisted reproduction technologies. Previous studies have focused on the use of vitamins $\mathrm{C}$ and $\mathrm{E}$ as potent antioxidants for semen cryopreservation in mammals [1,2]. However, some polyphenols such as quercetin, a secondary metabolite in plants, have been found to exhibit higher antioxidant activity and lower toxicity than synthetic antioxidants $[3,4]$. Plant derived compounds and their capacity for improvement of fertility have been previously examined through intake as dietary supplements in humans and in animals [5,6]. Assisted reproduction technologies, such as cryopreservation and in vitro fertilization (IVF), may also involve the use of antioxidant supplements in culture media $[7,8]$.

Cryopreservation of semen consists of storing living spermatozoa at a very low temperature. This technique allows living cells to be stored for a long period before use in IVF [9]. Although sperm cryopreservation is a useful technique, it can affect sperm quality parameters, such as morphology, motility, viability and DNA integrity, through cryoinjury [10]. Cryopreservation of human semen produces reactive oxygen species (ROS), which may cause important damage in sperm cells. Spermatozoa were the first type of cells to be cryopreserved. However, improvements in cryopreservation are still needed since semen in infertile males often exhibits low sperm survival rates [11].

Most studies on plant derived antioxidants and their use in cryopreservation have been done on animals such as rams and bulls $[12,13]$. This review examines the recent advances in the use of three polyphenolic substances produced by plants, quercetin, catechins and resveratrol, and their potential utility in human semen cryopreservation. Studying the effects of natural plant derived antioxidants can lead to new insights for the development of assisted reproduction techniques and for the improvement of male fertility [7].

\section{Role of antioxidants in semen protection from reactive oxygen species}

ROS include oxidative radicals such as hydroxyl radicals $(\mathrm{OH})$ and non radical species such as hydrogen peroxide (H2O2). Both species are byproducts of normal cellular metabolism [5]. Free radicals, which are formed during energy production, are oxygen molecules with at least one unpaired electron. Their chemical structure makes them strongly reactive in the presence of amino acids, lipids or nucleic acids [14]. ROS are required in low 
concentrations for normal cellular functions. Excess production of ROS is controlled by natural antioxidants. Leukocytes and immature spermatozoa are the most important producers of ROS in semen. Spermatozoa produce ROS since they are required for a number of functions. For example $\mathrm{H} 2 \mathrm{O} 2$ is required for capacitation, a process that allows spermatozoa to bind to the zona pellucida of an oocyte and to fertilize the egg. Capacitation, hyper activation and sperm-oocyte binding are all cellular processes that require adequate levels of ROS $[5,14]$.

Semen produces a number of enzymatic antioxidants which defend spermatozoa from oxidative stress. Superoxide dismutase and glutathione peroxidase are the main antioxidants that ensure reduction of hydrogen peroxide to water and alcohol. Mature sperm, which may be more sensitive because of their high concentration of membrane unsaturated lipids, are protected from oxidative stress by the seminal plasma. Indeed, the seminal plasma contains high concentrations of antioxidants such as superoxide dismutase, xanthine oxidase, nitric oxide, catalase, glutathione peroxidase, ascorbic acid, thiols, uric acid, alpha-tocopherol and glutathione [15]. Nonenzymatic antioxidants, which act as scavengers of ROS, include vitamins $\mathrm{C}$ and $\mathrm{E}$, glutathione, amino acids as well as other compounds used as dietary supplements $[15,16]$.

Excess amounts of ROS can affect semen characteristics such as sperm concentration, morphology and motility. Infertility in men may be influenced by several factors, including medical, dietary and genetic factors. ROS can also cause DNA damage and cell death in sperm. Sperm membranes are also sensitive to oxidative damage, since they are made up of high levels of polyunsaturated lipids, which react with ROS. Lipid peroxidation in the membrane can cause deformations, impaired motility, DNA fragmentation and cell death. An imbalance between oxidant and antioxidant levels may cause oxidative stress in sperm [5,14]. Antioxidants have been used in a number of studies to counter oxidative stress, as dietary supplements in humans and in animals and as components of culture media in in vitro experiments [1]. During the past years, researchers have shown a growing interest in the antioxidant properties of plant derived substances on semen quality in animals and in humans [16].

\section{Activity of Polyphenols As Antioxidants}

Polyphenols may act as free radical scavengers and sometimes, as metal chelates [3]. Zhong and Zhou [4] emphasized the need to study bioactive compounds in plants and to understand their chemical structure and their mechanism of action. Recent studies have examined the effect of dietary polyphenols on semen quality and antioxidant activity $[6,17]$. This review examines the inclusion of polyphenols in assisted reproduction techniques, more specifically in semen cryopreservation for in vitro fertilization.

\section{Quercetin}

Quercetin is a dietary flavonoid produced by plants. It is known to have antiviral, anti-inflammatory and antioxidant properties [10]. This reddish pigment is found in many foods such as apple skin, red wine and red onions [18]. Quercetin acts as an antioxidant by scavenging free radicals and chelating divalent cations. This compound might have a role in reducing hydrogen peroxide, increasing sperm antioxidant defenses and preventing DNA damage induced by oxidative stress [10]. The antioxidant properties of flavonoids like quercetin can be explained by their chemical structure. The number and the position of the hydroxyl group in flavonoids influence antioxidant activity. However, it is important to note that flavonoids exhibit antioxidant activity at low concentration, but may cause cytotoxicity and cell death at high concentration. Several studies have focused on quercetin as a potent antioxidant for semen quality [7].

Studies have shown that quercetin can improve the quality of ram and stallion sperm after cryopreservation. Quercetin can also increase the longevity of spermatozoa in fresh semen by inhibiting glycolysis. Quercetin can prevent ROS formation through both enzymatic and nonenzymatic cellular systems such as NADPH oxidase and NADPH-dependent oxide-reductase [12]. Ardeshirnia et al. [19] have studied the effects of quercetin on the fertility of frozen ram spermatozoa. Their results show that the viability of frozen-thawed spermatozoa and the mean number of zygote, morula, and blastocyst stage embryos were increased by using 5 and $10 \mu \mathrm{g} / \mathrm{mL}$ quercetin in the freezing extender. This study also shows that viability, motility and fertility of spermatozoa decreased when higher concentrations, over $20 \mu \mathrm{g} / \mathrm{mL}$, of quercetin were used [19]. Thus, the effects of quercetin are dose dependent.

Zribi et al. [10] tested the effects of quercetin on human sperm survival and DNA integrity during cryopreservation and thawing by adding the antioxidant in the cryoprotective medium. They noted that a concentration of $50 \mu \mathrm{M}$ improved sperm motility and viability and also decreased sperm DNA damage after thawing. The authors also suggest testing a larger number of samples with different concentrations to examine the dose dependent effects of quercetin on human sperm cryopreservation [10].

Although flavonoid compounds exhibit strong antioxidant properties in vitro, their effects are minimized in vivo due to their low water-solubility, weak absorption and lower bioavailability. In another study on human spermatozoa, Moretti et al. [7] developed a particular strategy to overcome this problem. They loaded quercetin into water-soluble liposomes, which act as carriers for the antioxidant. They compared the potential of quercetin-loaded liposomes and of quercetin alone in preventing oxidative damage in human sperm. Results show a dose-dependent effect of quercetin on sperm viability and motility. Quercetin-loaded liposomes showed lower toxicity for sperm viability and motility than quercetin alone. However, quercetin administered through liposomes increased genotoxicity in DNA at high concentrations. The authors suggest that this negative effect may be due to the particular mechanism of action of liposomes, which deliver quercetin directly inside the sperm cell and affect DNA. Although quercetin alone appeared more effective at reducing oxidative stress than liposomes, the authors suggest further investigations on the use of liposomes to carry compounds into human sperm cells [7]. 


\section{Catechins}

Catechin polyphenols are the most important bioactive components in green tea. Their antioxidant activity is 20 times higher than that of vitamin C. These flavonoid compounds can reduce ROS levels by quenching free radical species and chelating transition metals [20]. Green tea catechins include epigallocatechin gallate (EGCG), epicatechin gallate (ECG), epicatechin (EC) and epigallocatechin (EGC) [21]. Some studies have focused on the effects of green tea extract on cryopreservation of ram semen, without describing the chemical components involved in semen protection [22]. Zhang and Zhou [4] emphasize the need to describe in detail the bioactive components of plant extracts and to understand their chemical structure and mode of action. Although Catechins exhibit strong antioxidant activity, more studies are required to understand their potential beneficial effects in human reproduction [20].

Green tea catechins can exhibit antioxidant activity. They can steal metal ions by the catechin moieties in polyphenol molecules. Their B-ring is the main site of antioxidant reactions. Their structure allows electron delocalization, hence their ability to quench free radicals. Although catechins do not all have the same ability in chelating metals, they share similar molecular structures. Although catechins have antioxidant properties, studies have also emphasized their pro-oxidant activity. As catechins are unstable, they can generate ROS through auto-oxidation. Reaction of EGCG and EGC with hydrogen peroxide causes oxidation in their A-ring, decarboxylation and formation of oxidation products. Thus, a hydroxyl radical is created and exhibits pro-oxidant activity. The stability of EGCG is dependent on several environmental variables, such as pH, oxygen level and temperature [20]. Moretti et al. [18] studied the effect of four flavonoids, epicatechin, rutin, naringenin and quercetin, on lipid peroxidation in swim-up selected human sperm. Although quercetin and rutin were potent antioxidants, epicatechin did not show any antioxidant activity at a concentration of $200 \mu \mathrm{M}$. The authors hypothesize that this result is due to the chemical structure of epicatechin. Since epicatechin is the only one among the four molecules that does not have a carbonyl group, its structure may influence antioxidant activity and make it less potent than quercetin [18].

Gadani et al. [23] studied the effect of different concentrations of EGCG in thawing extender for boar sperm after cryopreservation. Indeed, thawing is a delicate step during which ROS can damage sperm cells because of rapid oxygen metabolism after a dormant metabolic stage. Adding EGCG to the thawing extender for 1 hour did not improve sperm viability. However, a higher penetration rate during fertilization was observed for all concentrations. Results show that the addition of different concentrations of EGCG to thawed spermatozoa for one hour did not affect sperm viability and acrosome integrity, but it significantly improved in vitro penetration rate [23].

Animal models have been used in studies to examine the potential of green tea catechins in improving semen quality
[20].Tissue culture medium 199 (TCM-199) is used to preserve reproductive cells. TCM-199 contains catechins EC, EGC, ECG and EGCG. A study by Barakat et al. [24] has shown that a green tea extract supplement in TCM-199 at $0.3 \mathrm{mg} / \mathrm{ml}$ improved in vitro maturation and embryo development of sheep cumulus oocyte complexes to blastocyst formation. Although some studies show effective antioxidant activity in animal tissues, more research is required to determine the potential of green tea catechins in human fertility [20].

\section{Resveratrol}

Resveratrol is a phytoalexin, or plant antibiotic, produced by leaf tissues when the plant defends against fungal infection or exposure to ultraviolet light. This polyphenol can be found in many plant species, but mainly in grapes and red wine. Heat and ultraviolet radiation can cause isomerization of trans-resveratrol into cis-resveratrol [25]. Resveratrol is a potent antioxidant and scavenger of superoxide, hydroxyl and metal-induced radicals. It can also prevent lipid peroxidation in cell membranes as well as DNA damage caused by ROS [26]. Although resveratrol is a stilbenoid and quercetin is a flavonoid, these two polyphenols have similar antioxidant effects. They can prevent ROS formation by enzymatic and non-enzymatic systems, such as NADPH-oxidase and NADH-dependent oxido reductase [3].

The effect of resveratrol on human semen has been studied during the past few years. Aitken et al. [27] analyzed the effects of several polyphenols on mitochondrial membrane potential, oxidative stress and DNA structure. Human spermatozoa were incubated with polyphenols in concentrations ranging from $25 \mu \mathrm{M}$ to $200 \mu \mathrm{M}$. At doses below $100 \mu \mathrm{M}$, resveratrol showed effective antioxidant functions and limited lipid peroxidation without causing mitochondrial damage. Although the effects of resveratrol were positive, this compound can inhibit the activity of topoisomerase, an enzyme involved in DNA replication and transcription. Thus, more studies are necessary to understand the benefits and the risks associated with the use of resveratrol to improve sperm quality [27].

Garcez et al. [11] studied the effects of resveratrol as a supplement in cryopreservation medium of human semen. They noted that although resveratrol was able to prevent lipid peroxidation, the effect was not dose dependent. A decrease in sperm motility was also observed in both fertile and infertile men. Resveratrol could not improve sperm motility. Although resveratrol could not restore motility of spermatozoa, this compound was able to prevent oxidative damage caused by cryopreservation of semen [11]. Longobardi et al. [13] conducted a study on the effect of resveratrol supplementation in semen extender for cryopreservation of buffalo sperm. Sperm quality parameters, including viability, motility, capacitation and fertilizing ability, were evaluated after thawing. Results show a dose-dependent effect of resveratrol on sperm capacitation status. There was a significant reduction in capacitation at higher concentrations. Supplementation of semen extender with resveratrol improved 
membrane integrity and antioxidant capacity. Moreover, IVF was carried out by using semen treated with $50 \mathrm{mM}$ resveratrol. The fertilization rate improved significantly compared with the control groups not supplemented with resveratrol. Thus, supplementation of $50 \mathrm{mM}$ resveratrol decreased capacitation, decreased oxidative stress and improved membrane stability and in vitro fertilizing ability [13]. Studies conducted on animals and on humans show both positive and negative effects of resveratrol as an antioxidant.

\section{Conclusion}

Quercetin, catechin and resveratrol are three polyphenols that have been the subject of recent experiments in cryopreservation of human semen and assisted reproduction technologies. The three substances can act as antioxidants and exhibit potential for sperm protection against oxidative stress. The studies reported in this review show positive effects as well as some negative effects of the polyphenols, especially at high doses. More research is required to understand the cellular mechanisms involved in antioxidant activity, to examine the dose dependent effects of polyphenols and to find the most effective dosage to be used in semen handling and preservation.

\section{Acknowledgment}

This work is financed by FEDER Funds through the AÇORES 2020 Operational Program under the BEMAP-ET project - "ACORES01-0145-FEDER-000026

\section{References}

1. Taylor K, Roberts P, Sanders K, Burton P (2009) Effect of antioxidant supplementation of cryopreservation medium on post-thaw integrity of human spermatozoa. Reprod Biomed Online 18(2): 184-189.

2. De Vasconcelos Franco JS, Faheem M, Chaveiro A, Moreira da Silva F (2016) Effects of $\alpha$-tocopherol and freezing rates on the quality and heterologous in vitro fertilization capacity of stallion sperm after cryopreservation. Theriogenology 86(4): 957-962.

3. Silva ECB, Cajueiro JFP, Silva SV, Soares PC, Guerra MMP (2012) Effect of antioxidants resveratrol and quercetin on in vitro evaluation of frozen ram sperm. Theriogenology 77(8): 1722-1726.

4. Zhong R, Zhou D (2013) Oxidative stress and role of natural plant derived antioxidants in animal reproduction. J Integr Agr 12: 1826-1838.

5. Wright C, Milne S, Leeson H (2014) Sperm DNA damage caused by oxidative stress: modifiable clinical, lifestyle and nutritional factors in male infertility. Reprod Biomed Online 28(6): 684-703.

6. Ly C, Yockell-Lelièvre J, Ferraro ZM, Arnason JT, Ferrier J, et al. (2015) The effects of dietary polyphenols on reproductive health and early development. Hum Reprod Update 21(2): 228-248.

7. Moretti E, Mazzi L, Bonechi C, Salvatici MC, Iacoponi F, et al. (2016) Effect of Quercetin-loaded liposomes on induced oxidative stress in human spermatozoa. Reprod Toxicol 60: 140-147.

8. Agarwal A, Nallella KP, Allamaneni S, Said TM (2004) Role of antioxidants in treatment of male infertility: an overview of the literature. Reprod Biomed Online 8(6): 616-627.

9. Jang TH, Park SC, Yang JH, Kim JY, Seok JH, et al. (2017) Cryopreservation and its clinical applications. Integr Med Res 6(1): 12-18.

10. Zribi N, Feki Chakroun N, Ben Abdallah F, Elleuch H, Sellami A, et al (2012) Effect of freezing-thawing process and quercetin on human sperm survival and DNA integrity. Cryobiology 65(3): 326-331.
11. Garcez ME, dos Santos Branco C, Venturin Lara L, Pasqualotto FF, Salvador M (2010) Effects of resveratrol supplementation on cryopreservation medium of human semen. Fertil Steril 94(6): 2118-2121.

12. Banday MN, Lone FA, Rasool F, Rashid M, Shikar A (2017) Use of antioxidants reduce lipid peroxidation and improve quality of crossbred ram sperm during its cryopreservation. Cryobiology 74: 25-30.

13. Longobardi V, Zullo G, Salzano A, De Canditiis C, Cammarano A, et al. (2017) Resveratrol prevents capacitation-like changes and improves in vitro fertilizing capability of buffalo frozen-thawed sperm. Theriogenology 88: 1-8.

14. Ko EY, Sabanegh Jr ES, Agarwal A (2014) Male infertility testing: reactive oxygen species and antioxidant capacity. Fertil Steril 2014;102(6): 1518-1527.

15. Moreira da Silva F, Marques A, Chaveiro A (2010) Reactive oxygen species: A double-edged sword in reproduction. Open Vet Sci J 4(9): 127-133.

16. Kedechi S, Zribi N, Louati N, Menif H, Sellami A, et al. (2017) Antioxidant effect of hydroxytyrosol on human sperm quality during in vitro incubation. Andrologia 49(1): e12595.

17. Zhao J, Jin Y, Du M, Liu W, Ren Y, et al. (2017) The effect of dietary grape pomace supplementation on epididymal sperm quality and testicular antioxidant ability in ram lambs. Theriogenology 97: 50-56.

18. Moretti E, Mazzi L, Terzuoli G, Bonechi C, Iacoponi F, et al. (2012) Effect of quercetin, rutin, naringenin and epicatechin on lipid peroxidation induced in human sperm. Reprod Toxicol 34(4): 651-657.

19. Ardeshirnia R, Zandi M, Sanjabi MR (2017) The effect of quercetin on fertility of frozen-thawed ram epididymal spermatozoa. S Afr J Anim Sci 47(2): 237-244.

20. Roychoudhury S, Agarwal A, Virk G, Cho CL (2017) Potential role of green tea catechins in the management of oxidative stress-associated infertility. Reprod Biomed Online 34(5): 487-498.

21. Wittayarat M, Ito A, Kimura T, Namula Z, Luu VV, et al. (2013) Effects of green tea polyphenol on the quality of canine semen after long-term storage at $5^{\circ} \mathrm{C}$. Reprod Biol 13(3): 251-254.

22. Mehdipour M, Kia HD, Najafi A, Dodaran HV, García-Alvarez 0 (2016) Effect of green tea (Camellia sinensis) extract and prefreezing equilibration time on the post-thawing quality of ram semen cryopreserved in a soybean lecithin-based extender. Cryobiology 73(3): 297-303.

23. Gadani B, Bucci D, Spinaci M, Tamanini C, Galeati G (2017) Resveratrol and Epigallocatechin-3-gallate addition to thawed boar sperm improves in vitro fertilization. Theriogenology 90: 88-93.

24. Barakat IA, Al Himaidi AR, Rady AM (2014) Antioxidant effect of green tea leaves extract on in vitro production of sheep embryos. Pak J Zool 46(1): 167-175.

25. Mohammadi RK, Arablou T (2017) Resveratrol and endometriosis: In vitro and animal studies and underlying mechanisms (Review). Biomed Pharmacother 91: 220-228.

26. Ourique GM, Finamor IA, Saccol EMH, Riffel APK, Pês TS, et al. (2013) Resveratrol improves sperm motility, prevents lipid peroxidation and enhances antioxidant defences in the testes of hyperthyroid rats. Reprod Toxicol 37: 31-39.

27. Aitken RJ, Muscio L, Whiting S, Connaughton HS, Fraser BA, et al. (2016) Analysis of the effects of polyphenols on human spermatozoa reveals unexpected impacts on mitochondrial membrane potential, oxidative stress and DNA integrity; implications for assisted reproductive technology. Biochem Pharmacol 121: 78-96. 


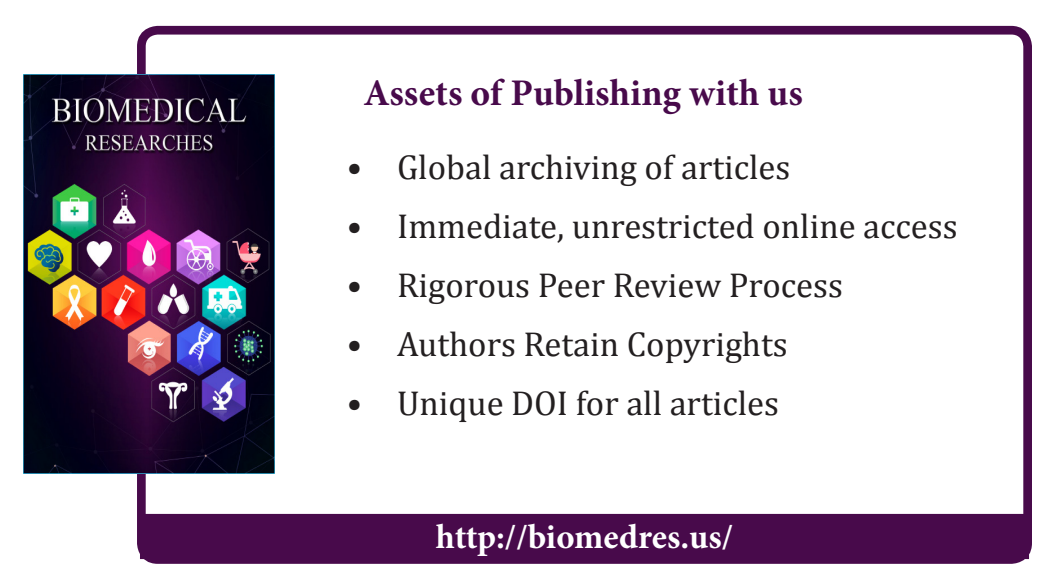

